\title{
Estratégias de gestão de risco de investimentos
}

\author{
Ronie Perterson Darid \\ DAMAT/UTFPR, Curitiba, PR \\ João Luis Gonçalves \\ DAMAT/UTFPR, Curitiba, PR \\ Maria Lúcia Abbott Jacolß \\ UTFPR, Curitiba, PR
}

\begin{abstract}
Resumo. Detalhamos a estrutura matemática dos métodos de Mínima Variância de Markowitz e Paridade de Risco utilizados em estratégias de gestão de risco de investimentos. Aplicamos essas estratégias na gestão de risco de uma carteira de investimento em ações de empresas brasileiras listadas na Bolsa de Valores de São Paulo, em um período que abrange os meses críticos da crise econômico-financeira causada pela pandemia de COVID-19. Os resultados indicam melhor desempenho destes métodos em comparação com a distribuição percentual uniforme de ativos. A estratégia de Mínima Variância apresentou resultados significativos na redução do risco, enquanto a estratégia de Paridade de Risco teve como principal aspecto positivo a manutenção da diversificação da carteira.

Palavras-chave: alocação de carteiras, mínima variância, equal weighting, paridade de risco, COVID-19.
\end{abstract}

\section{Introdução}

O ano 2020 ficará marcado na história mundial pelos grandes impactos sociais e econômicos causados pelo novo coronavírus, o SARS-CoV-2. A COVID-19 é a doença infecciosa causada por esse vírus, identificado em dezembro de 2019 após a notificação de um surto em Wuhan, na China, de acordo com a Organização Pan-Americana da Saúde (OPAS). A doença se espalhou pelo mundo rapidamente fazendo com que a Organização Mundial da Saúde (OMS) declarasse pandemia em 11 de março de 2020. As medidas tomadas após a declaração da pandemia tiveram reflexos econômicos no mundo todo, gerando uma crise global no mercado de ações e impactando fortemente as bolsas de valores. O índice Bovespa (Ibovespa), por exemplo, fechou o mês de março de 2020 com queda acumulada de $36,86 \%$ no ano.

As grandes perdas nos mercados acionários causadas pela pandemia de COVID-19 evidenciaram uma vez mais a importância das estratégias de gestão de risco de carteiras de investimento. Portanto, é relevante a reanálise de estratégias que promovam redução de risco, que possam ser aplicadas sem grandes dificuldades técnicas e sem ter de arcar com os elevados custos de manutenção de ativos de proteção, como opções de venda.

Estratégias de gestão de carteiras baseadas nos métodos de Mínima Variância de Markowitz (MV) e da Paridade de Risco (PR) mostraram bons resultados 2 quando comparados com a distribuição uniforme dos ativos na carteira (estratégia EW), durante a crise de 2008.

\footnotetext{
${ }^{1}$ ronie@utfpr.edu.br

2 jlgoncalves@utfpr.edu.br

3 mlajacob@yahoo.com.br
} 
Ambos os métodos tratam risco em termos da variância dos seus retornos e visam reduzi-lo através da determinação adequada dos pesos de cada ativo na composição da carteira.

No pioneiro artigo Portfolio Selection [9], Harry Markowitz propôs o modelo média-variância, no qual assumem-se as hipóteses de racionalidade do investidor e da eficiência dos mercados. Nesse modelo, buscam-se as carteiras eficientes, nas quais para um determinado risco tem-se o maior retorno. O conjunto das carteiras eficientes é denominado Fronteira Eficiente. Do modelo médiavariância decorre o método MV, que busca a alocação dos ativos na carteira visando somente minimizar o risco.

O método de Paridade de Risco (PR) utiliza os mesmos conceitos da média-variância e também visa a redução de risco, mas atribuindo os percentuais dos ativos na carteira de maneira que o risco de cada ativo contribua igualmente no risco total da carteira. A estrutura matemática do método foi apresentada em [8] e os trabalhos 2211] consideraram aplicações ao mercado brasileiro. Embora os métodos de Markowitz sejam os mais utilizados pelo mercado, o método PR tem ganho relevância pois há grandes fundos de investimentos que o utilizam.

Neste trabalho, analisamos o desempenho de duas estratégias de redução de risco de uma carteira de investimentos durante a crise causada pela COVID-19, uma através do método MV e a outra através do método $\mathrm{PR}$, ambas rebalanceando semanalmente a carteira.

O período analisado foi de janeiro de 2019 a agosto de 2020. A periodicidade escolhida foi semanal, tanto para o cálculo dos retornos que compõem as matrizes de covariância quanto para o rebalanceamento das carteiras.

Os resultados obtidos usando as diferentes formas de gestão refletiram a teoria que foi apresentada e validam a eficiência das estratégias no que se propõem.

\section{Métodos de gestão de risco de investimento}

Uma carteira de investimentos é um conjunto finito de ativos financeiros, tais como ações, opções, títulos, etc..., e seus respectivos pesos. Suponha que uma carteira $x$ é formada pelos ativos $1, \ldots, n$. O peso do ativo $i$ em $x$ é definido como o percentual do capital da carteira alocado em $i$ e é denotado por $x_{i}$. Desta forma, a carteira $x$ é representada pelo vetor

$$
x=\left(x_{1}, \ldots, x_{n}\right), \text { com } x_{1}+\ldots+x_{n}=1 \text {. }
$$

Uma estratégia de investimento é uma sequência de carteiras. Cada carteira de uma estratégia é obtida pelo rebalanceamento da carteira anterior. Por exemplo, a estratégia Equal Weighting (EW), também conhecida como $1 / n$, consiste na divisão igualitária do capital disponível entre os $n$ ativos da carteira, isto é, os pesos são

$$
x_{i}=\frac{1}{n}, \text { para } i=1, \ldots, n .
$$

Em um dado período, o retorno da carteira $x$ composta por $n$ ativos, com respectivos retornos $R_{1}, \ldots, R_{n}$, é dado por

$$
R(x)=\sum_{i=1}^{n} R_{i} x_{i} .
$$

O risco de um ativo é definido pelo desvio padrão dos seus retornos em relação ao retorno esperado, o qual será estimado neste trabalho pela média dos retornos históricos do ativo em um dado período. Por sua vez, o risco da carteira leva em conta a covariância entre os retornos dos ativos. Para $i \neq j$, seja $\sigma_{i j}=\operatorname{cov}(i, j)$ a covariância entre os retornos dos ativos $i$ e $j$ da carteira 
$x=\left(x_{1}, \ldots, x_{n}\right)$ e $\sigma_{i i}$ a variância dos retornos do ativo $i$. O risco da carteira de $x$ é definido por

$$
\sigma(x)=\left[\sum_{i=1}^{n} \sum_{j=1}^{n} x_{i} x_{j} \sigma_{i j}\right]^{1 / 2}=\left[x M x^{T}\right]^{1 / 2},
$$

em que $M$ é a matriz de covariância, isto é, $M_{i j}=\sigma_{i j}$. O risco da carteira depende essencialmente da matriz de covariância, que por sua vez depende da escolha dos ativos, dos dados históricos e pode variar conforme o período e a periodicidade.

\subsection{O Método de Mínima Variância de Markowitz}

O Método de Mínima Variância (MV) busca minimizar o risco e resulta na carteira de mínima variância, que corresponde ao ponto de menor risco na Fronteira Eficiente. Denotando $\mathbf{1}=$ $(1, \ldots, 1) \in \mathbb{R}^{n}$, o MV pode ser definido como o problema de otimização

$$
\min _{x \in \mathbb{R}^{n}} \frac{1}{2} x M x^{T} \text { s.a } 1 x^{T}=1 \text { e } x_{i} \geq 0, i=1, \ldots n
$$

No Problema 5 a função objetivo é quadrática e limitada inferiormente por zero, pois $M$ é simétrica e semi definida positiva. Além disso, as restrições de igualdade e de desigualdade são lineares e, em particular, as restrições de desigualdade são convexas.

A existência de um minimizador global para o Problema 5 segue do resultado apresentado em [4] e [1], e cuidadosamente demonstrado em [7, Teorema 1.34]. Como a função objetivo em (5) é contínua em $\mathbb{R}^{n}$ e as restrições formam um conjunto compacto, a existência de solução para o Problema 5 também segue do Teorema de Weierstrass [6. Corolário 2.5-7, pg. 81]. A supressão das restrições de desigualdade corresponderia a permitir vendas a descoberto na carteira. Neste caso, a solução pode ser explicitada conforme apresentado em [3, Proposição 5.9].

\section{$2.2 \quad$ A Paridade de Risco}

Nos métodos MV e EW cada ativo contribui de forma irregular para o risco da carteira. No trabalho [8] foi estruturado o método de Paridade de Risco (PR), cujo objetivo é obter carteiras em que todos os ativos possuem a mesma contribuição para o risco. Na prática, o PR produz carteiras diversificadas e riscos intermediários entre os métodos MV e EW. Os trabalhos [11] e [2] analisam estratégias baseadas no método PR no mercado brasileiro.

Definimos o risco marginal do ativo $i$ como a derivada parcial da função variância (4) com relação ao peso $x_{i}$,

$$
\frac{\partial \sigma(x)}{\partial x_{i}}=\frac{1}{\sigma(x)} \sum_{j=1}^{n} x_{j} \sigma_{i j}
$$

e a contribuição de risco do ativo $i$ como $\sigma_{i}(x)=x_{i} \frac{\partial \sigma(x)}{\partial x_{i}}$. O risco total da carteira é a soma das contribuições de risco dos ativos, conforme o Teorema 2.1, apresentado em [8].

Teorema 2.1. A função $\sigma$ decompõe-se como $\sigma(x)=\sum_{i=1}^{n} \sigma_{i}(x), x \in[0,1]^{n}$.

Sua demonstração é uma aplicação direta do Teorema de Euler para funções homogêneas 12 , p.34], conforme segue. Dado um número inteiro $m$, uma função $f: \mathbb{R}^{n} \rightarrow \mathbb{R}$ é chamada homogênea de grau $m$ se $f(t x)=t^{m} f(x)$, para todo $x \in \mathbb{R}^{n}$ e todo $t \in \mathbb{R}$. Restringindo a definição para $t>0$, $f$ é dita positivamente homogênea de grau $m$, como é o caso da função $\sigma$ com $m=1$. 
Teorema 2.2 (Euler). Se $f: \mathbb{R}^{n} \backslash\{0\} \rightarrow \mathbb{R}$ é uma função positivamente homogênea e continuamente diferenciável de grau $m \geq 1$, então $f(x)=\frac{1}{m} \sum_{i=1}^{n} x_{i} \frac{\partial f}{\partial x_{i}}(x), \forall x=\left(x_{1}, \ldots, x_{n}\right) \in \mathbb{R}^{n} \backslash\{0\}$.

Demonstração Dado $x \in \mathbb{R}^{n} \backslash\{0\}$, definimos $g(t)=f(t x), t \in \mathbb{R} \backslash\{0\}$. Pela Regra da Cadeia, temos $g^{\prime}(t)=\sum_{i=1}^{n} x_{i} \frac{\partial f}{\partial x_{i}}(x)$. Por outro lado, temos $g(t)=t^{m} f(x)$ e agora $g^{\prime}(t)=m t^{m-1} f(x)$. O resultado segue calculando $g^{\prime}(1)$ nas duas fórmulas para a derivada de $g$.

A paridade de risco busca pesos na carteira de modo que para $i \neq j$, tenhamos

$$
\sigma_{i}(x)=x_{i} \frac{\partial \sigma(x)}{\partial x_{i}}=x_{j} \frac{\partial \sigma(x)}{\partial x_{j}}=\sigma_{j}(x) .
$$

De acordo com 8, impor a paridade de risco equivale a resolver o seguinte problema de otimização

$$
\min _{x \in \mathbb{R}^{n}} \sum_{i=1}^{n} \sum_{j=1}^{n}\left(\sigma_{i}(x)-\sigma_{j}(x)\right)^{2}, \quad \text { s.a } 1 x^{T}=1 \text { e } x_{i} \geq 0, i=1, \ldots, n .
$$

O Problema 6 tem função objetivo quadrática (variância das contribuições de risco) e restrições lineares. Portanto cabe exatamente a mesma análise aplicada ao Problema 5 quanto a existência de solução global. O valor mínimo da função objetivo deve ser zero.

\section{$3 \quad$ Metodologia e Resultados}

Vamos comparar o desempenho de três estratégias de investimento, cujos pesos dos ativos serão definidos pelos métodos de Mínima Variância (Subseção 2.1), Paridade de Risco (Subseção 2.2) e Equal Weighting, conforme (2). Para a resolução dos problemas de otimização, referentes aos métodos de Mínima Variância e Paridade de Risco, utilizamos o software Matlab.

Escolhemos 12 ações (Tabela 1) entre aquelas que compunham o índice Ibovespa em janeiro de 2019, e que representam os diversos setores da economia classificados pela própria $\mathrm{B}^{3}$.

Tabela 1: Ativos utilizados.

\begin{tabular}{c|c|c|c|c|c} 
N. & Ticker & Nome & N. & Ticker & Nome \\
\hline 1 & ABEV3 & Ambev S.A. & 7 & RADL3 & Raia Drogasil S.A. \\
\hline 2 & EQTL3 & Equatorial Energia S.A. & 8 & RENT3 & Localiza R.C. S.A. \\
\hline 3 & IGTA3 & Iguatemi E. S. S.A. & 9 & SBSP3 & SABESP S.A. \\
\hline 4 & ITUB4 & Itaú Unibanco S.A. & 10 & VALE3 & Vale S.A. \\
\hline 5 & MGLU3 & Magazine Luiza SA & 11 & VIVT4 & Telefônica Brasil S.A. \\
\hline 6 & PETR4 & PETROBRÁS S.A. & 12 & WEGE3 & WEG S.A.
\end{tabular}

Utilizamos os retornos semanais das ações, sem considerar ajustes por lucros e dividendos, taxas de negociação ou impostos.

As matrizes de covariância que consideramos são construídas calculando a covariância entre os retornos semanais dos ativos nas 52 semanas anteriores ao rebalanceamento das carteiras. Analisamos 87 semanas. A primeira delas é a primeira semana de janeiro de 2019. A semana 87 
corresponde à última semana de agosto de 2020. As matrizes de covariância são semi definidas positivas, ou seja, seus autovalores são não negativos. Isso é importante para a convexidade da função objetivo do Problema 5. Observe que utilizamos apenas dados anteriores ao momento dos ajustes das carteiras (out-of-sample).

Na Tabela 2 destacamos os retornos e riscos das estratégias nas semanas de 61 a 64, correspondentes ao período de 23 de fevereiro a 20 de março de 2020, período de maior impacto da crise.

Tabela 2: Retornos e riscos obtidos pelas três estratégias.

\begin{tabular}{c|rrrr|rrr} 
Semana & \multicolumn{5}{|c|}{ Retornos } & \multicolumn{3}{c}{ Riscos } \\
\hline & EW & MV & PR & IBOV & EW & MV & PR \\
\hline $\mathbf{6 0}$ & 0,62 & $-0,93$ & 0,21 & $-0,61$ & 2,19 & 1,61 & 1,98 \\
$\mathbf{6 1}$ & $-8,81$ & $-6,82$ & $-8,44$ & $-8,37$ & 2,18 & 1,62 & 1,99 \\
$\mathbf{6 2}$ & $-2,04$ & 2,47 & $-1,14$ & $-5,93$ & 2,52 & 1,86 & 2,32 \\
$\mathbf{6 3}$ & $-15,71$ & $-11,10$ & $-14,59$ & $-15,63$ & 2,55 & 1,87 & 2,32 \\
$\mathbf{6 4}$ & $-15,21$ & $-4,17$ & $-13,25$ & $-18,88$ & 3,39 & 2,23 & 3,00 \\
$\mathbf{6 5}$ & 8,99 & $-4,11$ & 5,03 & 9,48 & 3,97 & 2,25 & 3,32 \\
\hline
\end{tabular}

Notamos que a menor exposição ao risco da estratégia MV resultou em uma menor queda nos retornos em comparação com as estratégias EW e PR.

Na Figura 1, são apresentados os retornos acumulados das estratégias ao longo das 87 semanas. Destacamos que no período anterior à crise, todas as estratégias têm um retorno muito próximo.

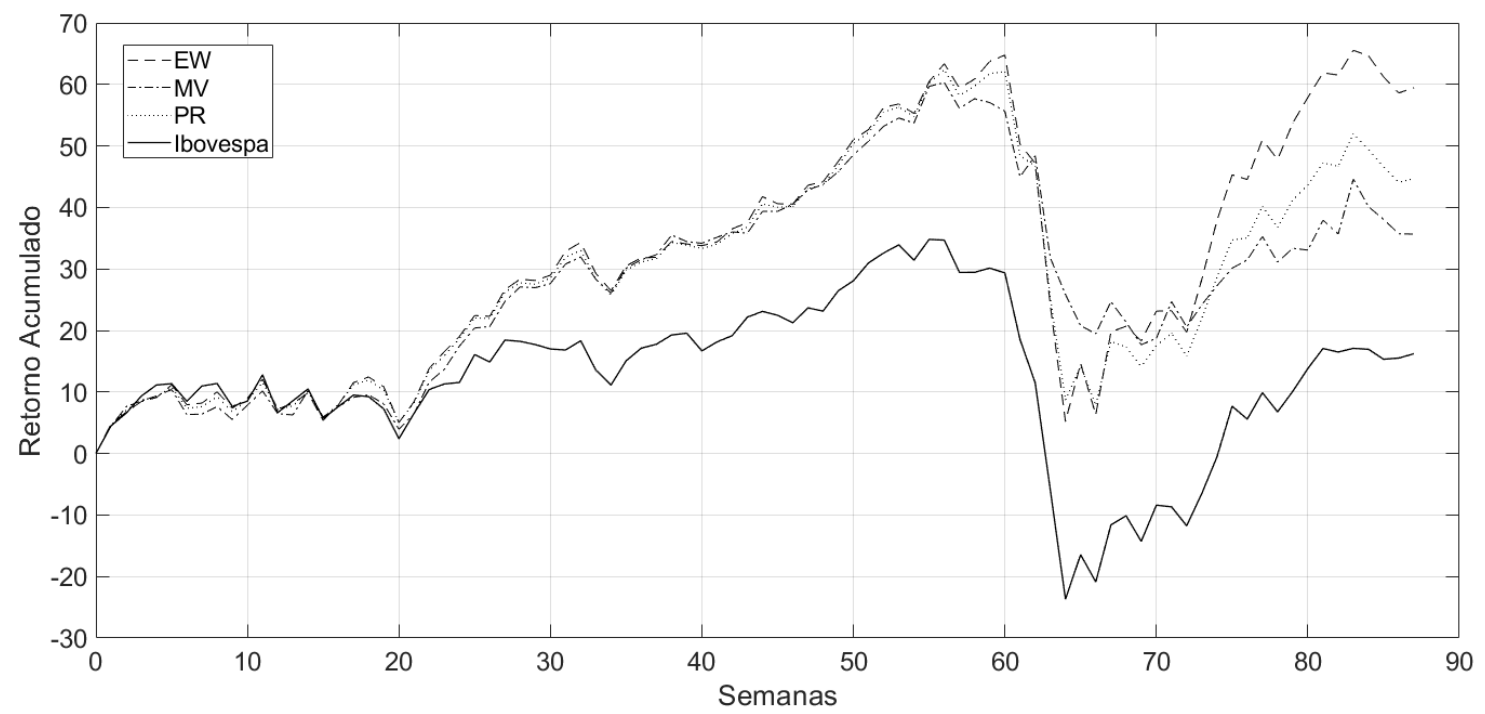

Figura 1: Retornos acumulados.

Nas semanas mais críticas da crise, naturalmente os retornos das três estratégias e do Ibovespa caem muito. Considerando o período do início da crise até a semana 87, o Ibovespa tem o piso do retorno acumulado em $-23,7 \%$. Já para as estratégias EW e PR os pisos foram 5, 2\% e 7, 8\%, respectivamente. A estratégia MV conseguiu amortecer melhor a queda causada pela crise e teve como piso do retorno acumulado $19,8 \%$.

No período posterior às semanas mais críticas, as estratégias EW e PR demonstram maiores retornos, superando a estratégia MV em retornos acumulados. Contudo, lembramos que a estratégia 
MV tem como único objetivo minimizar riscos e não impõe qualquer objetivo de performance quanto às taxas de retorno.

O risco estimado de cada estratégia é apresentado na Figura 2, Observamos que os riscos estão de acordo com a teoria 8, Apêndice A.3], que prevê que o risco da estratégia MV é menor ou igual que o da PR, que por sua vez é menor ou igual que o risco da EW. O risco da estratégia MV foi, em média, $88 \%$ do risco da estratégia $\mathrm{PR}$ e $81 \%$ do risco da estratégia $\mathrm{EW}$ no período anterior a crise, até a semana 60 .

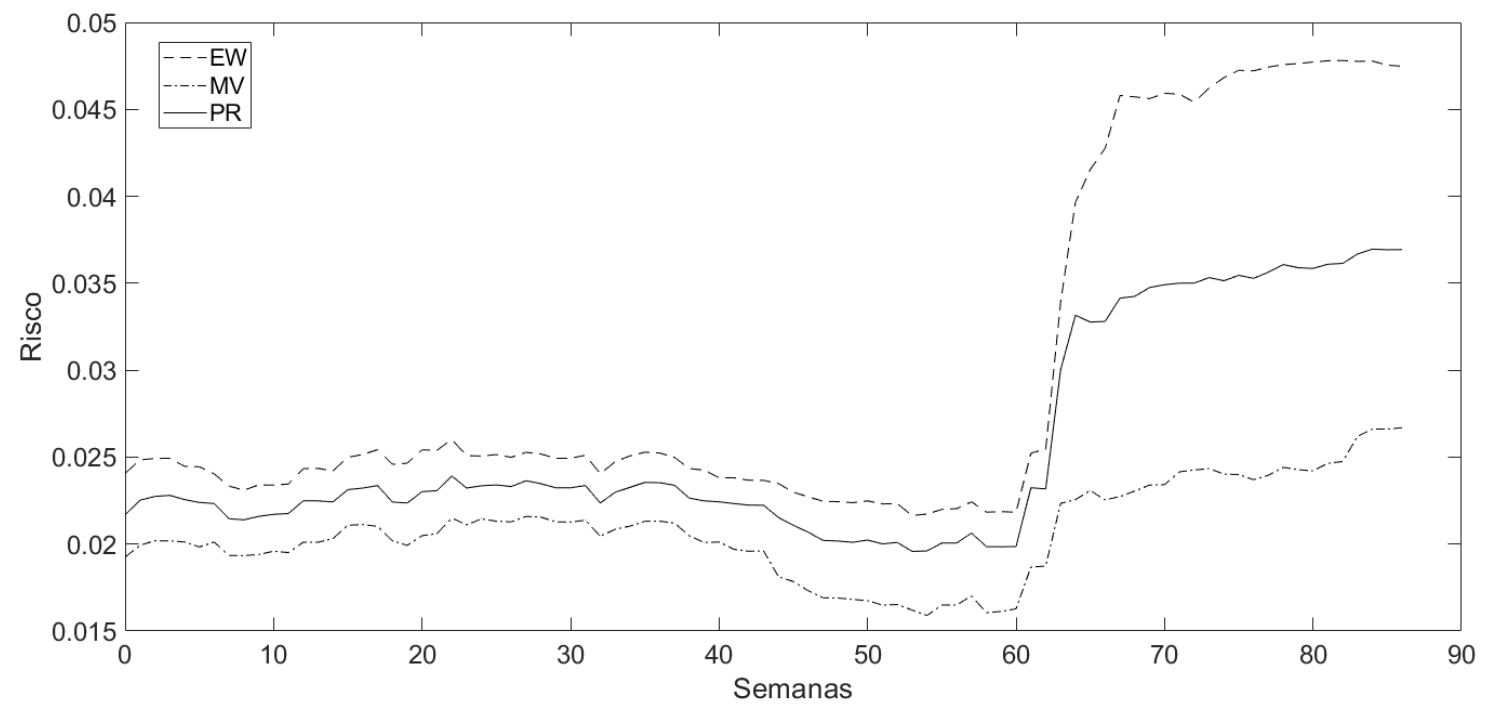

Figura 2: Risco.

A partir da semana 61 , o risco da estratégia MV passa para $70 \%$ do risco da estratégia PR e $55 \%$ da estratégia EW. Devido à crise, o risco aumenta em todas as estratégias. Porém, relativamente à estratégia $\mathrm{PR}$, o risco da estratégia MV diminui. Em relação à estratégia EW, o risco da estratégia MV aumenta pouco. Os resultados apresentaram os comportamentos que esperávamos das estratégias. Em particular, a estratégia MV conseguiu reduzir muito o risco e amortecer as quedas de retornos causados pela crise em comparação com as demais estratégias.

No período mais crítico da crise, as estratégias MV e PR identificaram os mesmos ativos como sendo os de menor risco e aumentaram os pesos dos mesmos. Embora a estratégia MV minimize o risco, ela concentra os investimentos em poucos ativos. Por outro lado, a estratégia PR mantém maior diversificação dos investimentos, mesmo durante a crise, como podemos ver na Tabela 3.

Tabela 3: Carteiras das estratégias MV e PR nas principais semanas da crise.

\begin{tabular}{c|c|cccccccccccc} 
& $\mathrm{t}$ & $x_{1}$ & $x_{2}$ & $x_{3}$ & $x_{4}$ & $x_{5}$ & $x_{6}$ & $x_{7}$ & $x_{8}$ & $x_{9}$ & $x_{10}$ & $x_{11}$ & $x_{12}$ \\
\hline $\mathrm{MV}$ & 61 & 5,67 & 23,41 & 0,00 & 0,07 & 0,00 & 0,00 & 16,66 & 3,10 & 0,00 & 17,23 & 33,87 & 0,00 \\
& 62 & 1,74 & 23,66 & 0,00 & 10,14 & 0,00 & 0,00 & 16,70 & 0,01 & 0,00 & 7,47 & 40,28 & 0,00 \\
& 63 & 0,07 & 22,99 & 0,00 & 12,39 & 0,00 & 0,00 & 17,24 & 1,83 & 0,00 & 8,43 & 37,03 & 0,00 \\
& 64 & 0,00 & 0,00 & 0,00 & 13,01 & 0,00 & 0,00 & 12,95 & 0,00 & 0,00 & 18,01 & 56,03 & 0,00 \\
\hline PR & 61 & 8,32 & 10,66 & 5,98 & 7,46 & 5,52 & 6,93 & 9,42 & 7,86 & 5,11 & 12,36 & 13,59 & 6,81 \\
& 62 & 8,35 & 10,75 & 6,24 & 9,70 & 5,59 & 6,51 & 9,75 & 7,77 & 5,39 & 10,10 & 13,56 & 6,30 \\
& 63 & 8,56 & 10,73 & 6,15 & 9,42 & 5,38 & 6,24 & 9,98 & 7,41 & 5,32 & 10,30 & 14,20 & 6,30 \\
& 64 & 7,78 & 8,07 & 5,61 & 9,60 & 6,90 & 4,84 & 8,76 & 6,54 & 6,55 & 13,67 & 15,98 & 5,70
\end{tabular}




\section{Conclusões}

Aplicamos as estratégias em um período que inclui a crise causada pela COVID-19 e assim testamos o desempenho das estratégias diante de grandes instabilidades para os investimentos. Os experimentos foram da forma out-of-sample e são completamente reproduzíveis.

Os resultados do método de Mínima Variância foram bastante positivos na propriedade para a qual ele foi desenvolvido, redução de risco. Assim como discutido nos resultados, a redução de risco converteu-se em menor queda nos retornos frente a momentos de grandes instabilidades. O comportamento de menor variância apresentado pela estratégia MV é vantajoso pois permite tomadas de decisão com maior perspectiva sobre a crise que se enfrenta.

A estratégia PR é intermediária quanto a gestão de risco, quando comparada com a MV e EW. Ela tem sido usada por alguns fundos de investimentos, por sua gestão de risco que, ao contrário da MV, preserva a diversificação, fato esse que pudemos verificar.

\section{Referências}

[1] Blum, E.; Oettli, W. Technical Note - Direct proof of the Existence Theorem for quadratic programming. Operations Research, Vol. 20, No. 1, p. 165-167, 1972. DOI: 10.1287/opre.20.1.165.

[2] Bortoluzzo, M., et al, Comparação do desempenho de carteiras utilizando os métodos paridade de risco, mínima variância e equal weighting: um estudo no mercado brasileiro em períodos pré, durante e pós a crise de 2008. Revista Evidenciação Contábil EG Finanças, Vol 6, No 3, p. 36-53, 2018. DOI: 10.22478/ufpb.2318-1001.2018v6n3.36822.

[3] Capinski, M., Zastawniak, T. Mathematics for Finance: An Introduction to Financial Engineering. Springer, 2006.

[4] Frank, M.; Wolfe, P. An algorithm for quadratic programming. Naval Research Logistics Quarterly, Vol. 3. p. 95-110, 1956. DOI: 10.1002/nav.3800030109.

[5] Jacob, M. L. A. Estratégias de gestão de risco de investimento no Brasil durante a pandemia de COVID-19. Dissertação de Mestrado, Universidade Tecnológica Federal do Paraná, Curitiba, 2021.

[6] Kreyzig, E. Introductory Functional Analysis with Applications. Jonh Wiley \& Sons, 1978.

[7] Krulikovski, E. H. M. Análise teórica de máquinas de vetores suporte e aplicação a classificação de caracteres. Dissertação de Mestrado, Universidade Federal do Paraná, Curitiba, 2017.

[8] Maillard, S.; Thierry, R.; Teileche, J. On the properties of equally-weighted risk contributions portfolios. The Journal of Portfolio Management, Vol. 36, No. 4, p. 60-70, 2010. DOI: 10.3905/jpm.2010.36.4.060.

[9] Markowitz, H. Portfolio selection The Journal of Finance, Vol. 7, No. 1, p. 77-91, 1952. DOI: 10.1111/j.1540-6261.1952.tb01525.x.

[10] Nocedal, J., Wright, S. J. Numerical Optimization. Springer, 2006.

[11] Souza, P. O. et al, Risk parity in the brazilian market Economics Bulletin, v. 37-3, p. 1555-1566. DOI: 10.2139/ssrn.3001878.

[12] Spivak, M. Calculus on Manifolds. Addison-Wesley Publishing Company, 1965. 